BULLETIN Bulletin hispanique

HISPANIQUE Université Michel de Montaigne Bordeaux

$122-2 \mid 2020$

L'épithalame en Espagne

\title{
Sátira menipea y renovación narrativa en España: del lucianismo a Don Quijote
}

Cordoue/Bordeaux: UCOPress/Presses Universitaires de Bordeaux, 2017

\section{Philippe Rabaté}

\section{(2) OpenEdition \\ Journals}

Edición electrónica

URL: http://journals.openedition.org/bulletinhispanique/11887

DOI: 10.4000/bulletinhispanique. 11887

ISBN: 1775-3821

ISSN: 1775-3821

\section{Editor}

Presses universitaires de Bordeaux

\section{Edición impresa}

Fecha de publicación: 20 diciembre 2020

Paginación: 762-767

ISBN: 979-10-300-0606-3

ISSN: 0007-4640

\section{Referencia electrónica}

Philippe Rabaté, «Sátira menipea y renovación narrativa en España: del lucianismo a Don Quijote», Bulletin hispanique [En línea], 122-2 | 2020, Publicado el 14 diciembre 2020, consultado el 19 enero 2021. URL: http://journals.openedition.org/bulletinhispanique/11887 ; DOI: https://doi.org/10.4000/ bulletinhispanique.11887

Este documento fue generado automáticamente el 19 enero 2021.

Tous droits réservés 


\title{
Sátira menipea y renovación narrativa en España: del lucianismo a Don Quijote
}

Cordoue/Bordeaux: UCOPress/Presses Universitaires de Bordeaux, 2017

\author{
Philippe Rabaté
}

\section{REFERENCIA}

Sátira menipea y renovación narrativa en España: del lucianismo a Don Quijote, Pierre DARNIS, Elvezio CANONICA, Pedro RUíz PÉREZ y Ana VIAN HERRERo (eds.). - Cordoue/Bordeaux: UCOPress/Presses Universitaires de Bordeaux, 2017, 344 p., col. Série Espaces Ibériques. ISBN 979-10-300-0105-1

1 Ce volume, coédité par la Universidad de Córdoba et par les Presses Universitaires de Bordeaux, est issu d'une rencontre scientifique qui s'est tenue à l'université Montaigne sur le thème de la satire ménippée et des survivances et réappropriations des œuvres de Lucien de Samosate dans les lettres du Siècle d'or. Cet ouvrage participe ainsi d'un renouveau des approches sur ce sujet complexe tant l'influence du lucianisme n'est connue que de manière parcellaire et parfois superficielle. Les 4 éditeurs de ce livre ont souhaité offrir ce volume en hommage à Michel Cavillac, professeur émérite de l'Université Michel de Montaigne, dont les travaux classiques sur Mateo Alemán s'appuient en partie sur la mise en lumière d'une trame ménippéenne dans le Guzmán de Alfarache. Toutefois, l'ouvrage ne porte pas principalement sur le récit alémanien (seules deux contributions lui sont consacrées) mais étend son périmètre d'investigation du premier humanisme - avec deux textes sur Cristóbal de Villalón et deux autres sur les Lazarillo de 1554 et 1555 - jusqu'à l'œuvre cervantine, abordée à trois reprises. Il est, de ce point de vue, regrettable que les bornes chronologiques ne soient pas davantage justifiées et que l'on ne dispose pas de contributions sur des 
œuvres du XVII ${ }^{e}$ siècle également influencées par ce type discursif comme celles de Francisco de Quevedo et de Baltasar Gracián.

Sátira menipea y renovación narrativa débute par une introduction dense de Pedro Ruíz Pérez qui explore la nature de la satire ménippée, entre genre littéraire, forme ou catégorie discursive. Pour ce faire, Pedro Ruíz Pérez nous invite à débuter notre lecture par deux noyaux du lucianisme du XVI ${ }^{\mathrm{e}}$ qui sont étudiés dans les contributions de Theodora Grigoriadu et de Folke Gernert, qui portent respectivement sur l'importance de Lucien dans les traductions humanistes espagnoles et sur le lucianisme chez quelques médecins castillans du XVI ${ }^{e}$. Ces deux corpus nous permettent d'aborder la satire ménippée à partir de la formation d'un discours humaniste, soit par le biais de l'adaptation en castillan, soit par le recours au modèle de l'éloge paradoxal (la tragodopodagra) chez les médecins Laguna et Jarava. Après ce premier contact avec la chair des textes, Pedro Ruíz Pérez aborde la question sensible de la nature de ce type de discours et prévient d'emblée son lecteur : «Desde la noción clásica de los genera y las teorías más actuales, difícilmente cabe concebir la sátira menipea como un género stricto sensu» (p.14) avant de préciser qu'il s'agirait davantage d'un «espacio discursivo no formalizado de manera cerrada». Aussi peut-on comprendre l'importance de la notion de tradition inséparable de toute considération générique - ce qui apparaît dans l'article de Nicolas Corréard - ainsi que la capacité d'hybridation des discours ménippéens, qui ressort clairement des articles de María José Vega et de Fabrice Quero. Il ne faut de la sorte jamais oublier, comme le rappelle Ana Vian, que la satire ménippée est satura mélange, ouverture, série indéfinie de traits - et de cette plasticité formelle témoignent également les articles de Miguel García Bermejo sur la Dolería et de Germán Redondo sur les dialogues d'Argensola. Si la satire ménippée est donc, de l'aveu même de Pedro Ruíz, un «discurso sin límites genéricos» (p. 15), cela n'interdit pas d'en dresser l'histoire.

3 Le dernier pan de son introduction est placé sous le signe d'une brève esquisse diachronique de la satire ménippée dans les lettres espagnoles qui voit se succéder trois époques distinctes: aux années 1520, marquées par la présence forte en Espagne de l'érasmisme et de la littérature autour du sac de Rome, succèdent deux décennies d'intense circulation des traductions de l'œuvre du Samosate et de productions fortement influencées par cette dernière. Dès le début des années 1550, la satire ménippée entame sa métamorphose avec le Viaje de Turquía et les deux Lazarillo, mue annonciatrice de la fin du XVI e siècle où coexistent des formes savantes et érudites de la ménippée, et des versions plus diluées, moins strictement fidèles à la facture lucianesque. Si Juste Lipse remplace Erasme comme référence de l'humanisme européen, la prose fictive cherche un nouvel équilibre entre delectare et prodesse, et la censure prend parfois des inflexions contre-réformatrices comme dans les Sueños de Quevedo, évoqués notamment par Nicolas Correard. Au terme de sa préface, Pedro Ruíz rappelle bien la prudence avec laquelle il convient de manier le terme même de novela en excluant toute vision téléologique.

4 Le corps du livre se décompose ensuite en cinq parties intitulées respectivement «Rasgos menipeos», «La sátira menipea en sus contextos», «¿Picaresca o Menipea?», «Cervantes lucianesco» et, enfin, «Varia para Michel Cavillac». Le premier ensemble de travaux nous conduit des traductions de Lucien en langue espagnole - fort nombreuses puisque Theodora Grigoriadu ne dénombre pas moins de 71 versions espagnoles issues de 13 traducteurs - à deux études précises sur $E l$ Crotalón de Cristóbal de Villalón, en passant par trois dialogues de Bartolomé Leonardo de Argensola (Germán Redondo Pérez) et la 
méconnue Comedia llamada Dolería del Mundo de Hurtado de la Vera dont la première édition remonterait à 1572 (étudiée par Miguel García-Bermejo Giner). Ces différents articles tendent à identifier des éléments ménippéens constitutifs dans les œuvres qu'ils étudient respectivement. Ana Vian se penche ainsi sur le voyage de Gallo vers le ciel et l'enfer dans $\mathrm{El}$ Crotalón en esquissant des liens convaincants avec la tradition de miseria hominis qui n'exclut par le recours à l'humour comme arme défensive et philosophique face aux nombreuses incertitudes dans lesquelles sont plongés les esprits conscients du premier XVI ${ }^{e}$ siècle. La contribution de Fabrice Quero s'attache à un épisode plus circonscrit, «la visita al palacio menipeo de la maga Saxe (cantos 5-7)». El Crotalón est une œuvre hybride, à la fois démonstrative et séduisante, rhétorique et historique, et le chant 6 permet ainsi à Gallo «en un mismo discurso, discantar y orar a través de la estricta superposición del relato ficcional sobre la crónica histórica» (p. 92). Les masques dont se sert l'auteur, la fine auto-ironie qu'il déploie sont l'un des traits les plus ménippéens du récit. Germán Redondo Pérez et Miguel García-Bermejo Giner s'attachent à étudier les traits ménippéens qui apparaissent dans les discours d'Argensola pour le premier et dans la la Comedia llamada Dolería del Mundo pour le second; cette dernière œuvre offre ainsi une vision typiquement lucianesque où la satire ménippée offre une sorte d'espace discursif, d'anatomía au sens le plus figuré.

5 La seconde partie de l'ouvrage, «la sátira menipea en sus contextos», commence par une contribution riche de María José Vega sur deux textes italiens importants, le Palinurus ( ca 1445) de Maffeo Veggio, où le pilote d'Enée tient le rôle principal, et sa réécriture par Antonio Brucioli dans le Dialogo della umana miseria (1526). La considération conjointe de ces deux dialogues invite María José Vega à relativiser l'influence de Lucien sur ces deux textes même si elle demeure indéniable, au profit d'une prise en considération de la tradition de miseria hominis qui se manifeste notamment dans la critique qui touche les différentes conditions sociales - en particulier celle des hommes de pouvoir, peu fréquente dans les œuvres sur la misère de l'homme. Le poids du stoïcisme est manifeste, ainsi que l'influence d'un traité pseudo-platonicien, l'Axiochus, ce qui suffit à prouver la variété des sources textuelles de ces œuvres. Pour sa part, Folke Gernert ébauche quelques éléments sur la référence à Lucien parmi les médecins humanistes, qui peut prendre la forme de lectures, de traductions ou d'adaptations de l'œuvre du Samosate comme la version latine du Tragodopodagra par Laguna ou l'Icaromenipo traduit en castillan par Juan de Jarava. Le dialogue est ainsi perçu comme un genre apte à rendre compte de la variété de la Nature.

Sònia Boadas propose pour sa part une étude des deux rédactions de la República literaria de Saavedra et de ses liens avec les fameux Ragguagli di Parnaso de Trajano Boccalini. Si la première version de l'œuvre - probablement rédigée entre 1615 et 1620 - s'inspire, tout comme l'œuvre italienne, de nombreux traits propres à la satire ménippée (ton satirico-burlesque, contexte fantastique de l'action, dialogue avec les morts et révisions critiques du canon littéraire), Sònia Boadas montre avec aisance combien la seconde version du texte alterne proximité et prise de distance avec l'œuvre boccalinienne, si bien que «la segunda redacción se aleja de la burla y de la crítica mordaz y acude al texto boccaliniano como fuente de preceptos políticos y lecciones morales (...)» (p. 169).

7 La troisième partie, sobrement intitulée «ipicaresca o menipea?», voit se succéder trois contributions qui portent, pour les deux premières, sur le Lazarillo et/ou ses continuations et, pour la troisième, sur le Guzmán de Alfarache. L'article de Valentín Núñez Rivera aborde l'héritage des œuvres de Lucien et d'Apulée dans le récit anonyme 
de 1554 et dans ses deux suites, publiées respectivement en 1555 et 1620 . Si le premier texte de la série offre un lucianisme quasiment imperceptible à cause du caractère vraisemblable, voire vériste, de la narration, la continuation de 1555 va en revanche jouer sur le caractère métamorphique des œuvres de Lucien et en développer tout le potentiel comme dans la fameuse rencontre avec la vérité (chapitre 15). Enfin, le texte de 1620 jouerait un rôle intermédiaire et proposerait une relecture des deux premiers textes à travers le prisme d'une réflexion sur la fiction. Pour sa part, la contribution d'Alfredo Rodríguez López-Vázquez propose de revenir sur l'hypothèse, vite écartée, d'une paternité commune pour les deux textes anonymes. Le spécialiste de la Segunda parte de Lazarillo de Tormes, qu'il a d'ailleurs éditée en 2014 (Cátedra, Letras Hispánicas), énumère ainsi plusieurs artifices que les deux œuvres partagent et qui sont, pour certains d'entre eux, d'inspiration ménippéenne: une intention satirique qui relève d'une «teología burlona» (la formule est de Juan de Pineda pour qualifier le premier Lazarillo), l'usage d'une trame biblique utilisée de manière subversive, les exemples introduits dans le corps du texte, les procédés qui relèvent de l'humour et d'une stratégie de dissimulation de la part de l'auteur et, enfin, les procédés rhétoriques. L'auteur conclut en affirmant que la première partie serait une «sátira menipea clásica» lorsque la seconde ressortirait à «sátira lucianesca». La contribution de Montserrat Jufresa vient clore cette partie picaresque en offrant un ensemble de réflexions sur le profil de Guzmán et développe plusieurs thèmes - l'éloge de la pauvreté, la vie selon la nature, la critique de la stupidité humaine et la liberté radicale de l'homme - qui dessinent de nombreuses influences dans le texte alémanien parmi lesquelles se détachent la source grecque et l'héritage du Momus d'Alberti. L'on peut regretter que la dialectique entre le pécheur et le juste éprouvé par Dieu ne soit pas davantage développée, mais l'inclination éthique et politique de Mateo Alemán pour le modèle florentin, que la conclusion rappelle, est indiscutable.

8 La quatrième partie, "Cervantes lucianesco», a pour objet l'importance des œuvres du Samosate avec un premier texte où Adrian J. Saéz explore la relation complexe que Cervantes établit avec la vraisemblance, et les jeux subtils qu'il ébauche entre vérité et mensonge. Si Lucien tend à défaire l'autorité de son narrateur, l'auteur du Don Quichotte s'ingénie davantage à multiplier les masques. La relation aux merveilles et prodiges retient également l'attention d'Adrian J. Saéz et l'on ne peut que remarquer la variété de la vision cervantine, de la «labor de desmontaje escéptico» face à l'épisode de la cueva de Montesinos (Don Quichotte, II, ch. 22-24) jusqu'à la représentation de la lycanthropie dans le Persiles, qui offre une considération bien différente de la merveille. En s'attachant à une lecture du Don Quichotte de 1605, Pierre Darnis offre une série de réflexions sur «la philosophie du gouvernement amoureux » dans le texte cervantin. Après avoir rappelé le lien souvent établi entre romans de chevalerie et lascivité, notamment pré-matrimoniale, l'auteur de cette contribution développe trois thèmes récurrents des discours sur l'amour: l'avertissement adressé aux «néophytes", les "conseils pour sortir du malheur" qui, bien souvent, conduisent à un mariage réparateur, et quelques éléments pour choisir le bon conjoint. Il en ressort une forme d'exemplarité propre à l'œuvre de Cervantes. Nicolas Correard développe pour sa part une approche du Colloque des chiens fondée sur la métamorphose souvent associée à la littérature ménippéenne. Fort d'une approche comparatiste, l'auteur de cette contribution dégage une forme de moralisation de la «cultura menipea» (p. 287) dans la nouvelle cervantine qui vise à en écarter les traits les plus scandaleux. Si Cervantes développe une poétique subtile de la crédulité et de l'incrédulité, il conduit également 
son lecteur à s'interroger sur les deux pôles du discours fictif que sont le don de la parole et la médisance. La satire énoncée par Scipion devrait être ainsi considérée dans sa dimension allégorique, en une sorte de fidélité à l'esprit érasmien.

Une dernière partie recueille deux articles d'anciens disciples de Michel Cavillac avec, tout d'abord, une contribution de Francis Desvois, auteur d'une traduction intégrale du Guzmán (Classiques Garnier, 2014). Dans un style alerte, Francis Desvois revient sur quelques points de sa traduction - titre, "proverbes, dictons, apophtegmes ", jeux de mots et, enfin, "questions lexicales diverses »- qui justifient la nécessité d'une nouvelle traduction. Anne Dubet revient pour sa part sur les métamorphoses de «La literatura de avisos», en proposant une relecture du phénomène arbitriste à la lumière de sa réception au XVIII ${ }^{e}$ siècle. L'auteur de ce texte, dont les études sur la littérature politique et fiscale castillanes font référence, remet en cause plusieurs idées ancrées dans les esprits, à commencer par celle qui ferait des arbitristes les fruits d'une décadence. Une comparaison avec le cas français suffit à nuancer une telle affirmation, et les arbitristes ne forment pas davantage un groupe à part si on lit leurs textes comme des «actos políticos» (p. 322). Il est indéniable que la monarchie prête attention à leurs écrits de manière croissante dans une stratégie politique de négociation avec les élites espagnoles. Sur un temps plus long, les proyectistas succèdent aux arbitristes avec le changement dynastique, et leur projet s'intègre davantage dans une économie politique qui promeut une discussion publique, loin du secret du pouvoir.

Les quelques éléments de présentation que nous venons de donner peinent à rendre compte de la richesse de ce volume qui constitue un bel hommage à l'œuvre importante de Michel Cavillac. On se prendrait à rêver que le voyage à travers les œuvres ménippéennes se poursuive, et qu'apparaisse alors une contribution supplémentaire sur les Exequias de la lengua castellana de Juan Pablo Forner, dont le sous-titre comporte également la mention «Sátira menipea». Un tel prolongement aurait également permis de rendre hommage aux pages magnifiques qu'un autre grand professeur bordelais, François Lopez, consacra à ce texte extraordinaire et corrosif dans sa très belle thèse, Juan Pablo Forner et la crise de la conscience espagnole.

\section{AUTORES}

\section{PHILIPPE RABATÉ}

Université Paris-Nanterre/IHRIM ENS de Lyon 